Cahiers d'études africaines

\title{
Livres de passages
}

Trajectoires migrantes vers et depuis Dakar

Books of Passageways. Migratory Paths to and from Dakar

\section{Catherine Mazauric}

\section{CpenEdition}

Journals

Édition électronique

URL : http://journals.openedition.org/etudesafricaines/17659

DOI : $10.4000 /$ etudesafricaines. 17659

ISSN : 1777-5353

Éditeur

Éditions de l'EHESS

Édition imprimée

Date de publication : 30 juin 2014

Pagination : 267-287

ISSN : 0008-0055

Référence électronique

Catherine Mazauric, "Livres de passages », Cahiers d'études africaines [En ligne], 213-214 | 2014, mis en ligne le 27 juin 2016, consulté le 19 avril 2019. URL : http://journals.openedition.org/ etudesafricaines/17659; DOI : 10.4000/etudesafricaines.17659 


\section{Livres de passages}

\section{Trajectoires migrantes vers et depuis Dakar}

Les mobilités internes à l'Afrique de l'Ouest précèdent de loin, historiquement, les migrations Sud-Nord. Sans nullement s'être taries pour autant, elles s'ancrent dans une temporalité au moins partiellement distincte de celle qui s'est construite à partir du moment colonial, puis post-colonial. Aujourd'hui, dans les discours des médias, mais aussi les productions scientifiques, la visibilité des secondes paraît cependant surpasser largement celle des premières, jusqu'à tendre à les occulter. Qu'en est-il des récits littéraires ? L'une des questions que l'on peut formuler à leur propos porte sur les éventuelles disjonctions ou continuités repérables entre les représentations qui ont été élaborées, pour l'Afrique de l'Ouest, de ces deux modalités du mouvement migratoire : une circulation interne au continent ou au sous-continent, dite Sud-Sud, ou un déplacement vectoriel intercontinental, dit Sud-Nord.

\section{Moment charnière, lieu capital}

Pour s'en tenir aux textes de langue française, parmi les ouvrages formant le canon littéraire ouest-africain, des récits de voyages, des scènes de départ et d'arrivée relatives à un déplacement, généralement ferroviaire, de la brousse à la capitale, sont bien connues : ainsi dans L'Enfant noir (Camara 1953), Maïmouna (Sadji 1958), ou encore Karim (Socé 1948). Significativement, ces récits et ceux qui pourront en être rapprochés ont été écrits et publiés dans la période d'une vingtaine d'années (entre 1937 et 1958) précédant la proclamation des Indépendances. Tandis que les personnages éponymes de Sadji et Socé découvrent, dans l'ébahissement, l'étourdissement et l'éblouissement mêlés, l'agitation «moderne » de Dakar, l' «enfant noir » désormais adolescent, au neuvième chapitre du roman autobiographique de l'auteur guinéen, s'en va à Conakry poursuivre ses études, et débuter ainsi une nouvelle phase de son existence. La capitale guinéenne ne constitue qu'un rouage de «l'engrenage » qui, «de l'école de Kouroussa, condui[t] à Conakry et abouti [t] à la France » (Camara $1953: 218)$. La première nuit que le narrateur y passe est aussi sa première « dans une maison européenne » (ibid. : 169); en la visitant, c'est sa différence d'avec Kouroussa qui le frappe, notamment des avenues « tirées au cordeau » et « se coup[ant] à angle 
droit » (ibid. : 170), représentative d'un urbanisme orthogonal, lui-même exhibant l'emprise du pouvoir colonial, dont les règles sont venues se superposer et s'imposer à ce qu'il a voulu percevoir comme un désordre originel. La capitale géométrisée forme, de manière linéaire et transparente, l'antichambre de la métropole dans le programme narratif du héros. Quant à Maïmouna, sa première vision de Dakar, au lendemain de son arrivée, est celle d'une «capitale des tropiques avec ses bâtiments que des conceptions nouvelles remaniaient chaque jour » (Sadji 1958: 86), entre le port où s'agitent «de longs bras métalliques, mûs par des treuils », « des cargos et des transatlantiques », et le ciel où « le danger plan[e]», «avec le vrombissement des avions, guetta[n]t le passant étourdi aux angles des carrefours » (ibid.). Dès le premier regard sur la ville, celle-ci est ainsi appréhendée comme un nœud du trafic intercontinental que figurent les machineries de la technologie moderne. Quant à l'identité de ce "fief de la civilisation", elle s'établit à travers la construction d'une série d'antithèses avec ce qui forme celle de la «brousse sénégalaise »: aux «bâtiments» en perpétuel remaniement s'opposent les "grandes savanes », aux "pâturages » et au « roc ancestral », « la dalle, le goudron et l'asphalte », aux marcheurs sans hâte, des «foules » qui «coulent dans les rues et fuient» (ibid.), etc. La périphérie urbaine apparaît alors comme une zone de contact, intermédiaire et précaire, entre horizontalité (celle des «grandes savanes » à présent recouvertes par «la dalle, le goudron et l'asphalte») et verticalité (des engins du port, des «bâtiments », des « quartiers neufs » qui « champignonnent»), figement et flux, décrépitude et vitalité, passé immobile et futur conquérant :

« Aux confins de la ville de pierre, les agglomérations indigènes s'étalaient, rousses et poussiéreuses. Comparées aux quartiers neufs, riants et pittoresques qui champignonnaient dans le centre, sur le plateau et sur le roc, ces agglomérations évoquaient par leur aspect sordide la misère et la décrépitude qui s'étalaient partout à l'intérieur du pays. Elles formaient comme une ceinture d'ordures qui s'élargissait à mesure que le flot grondant de l'urbanisme déferlait sur elle» (ibid.).

La ville forme une jonction entre l'espace, confus, indifférencié et pratiquement moribond, de la brousse, et le monde régulé, ordonné, cependant plein d'énergie vitale, de l'ailleurs «blanc». Mais le dynamisme de celuici, communiqué à la presqu'île dakaroise, mue celle-ci en ogresse dévoratrice de ce passé englouti, tout autant qu'il anime le mouvement brownien des artères de la capitale. La jonction spatiale est aussi disjonction temporelle.

Quelques années plus tôt et sur un autre rythme, Ousmane Socé Diop avait fait découvrir le cœur de Dakar-Plateau (Hôtel de Ville, avenue WilliamPonty) à un Karim en calèche, sensible aux beautés de l'architecture coloniale et à l'élégance des passantes, tant africaines qu'européennes : « Karim était rempli d'admiration pour Dakar, la ville jeune, moderne, un prolongement de la métropole » (Socé 1948 : 70). Dans Mirages de Paris, du même Ousmane Socé Diop (1964 [1937]), un texte encore antérieur, l'étape dakaroise avait été pratiquement omise. Mais lorsque Sadji fait découvrir le centre 
de Dakar à travers le regard de la jeune Maïmouna, il retrouve les accents d'un Socé montrant le personnage principal de Mirages de Paris, Fara, ébaubi, à Paris, devant « ces millions d'hommes qui marchent inlassablement », «l'habileté des parisiens qui se faufilent entre les voitures », " mécaniques et hommes se coudoya[n]t avec souplesse », " maisons plus hautes », etc. (ibid. : 29).

De manière plus nette encore que la scène de départ, la scène d'arrivée forme ainsi un topos commun, littérairement transmis et donc objet d'une intertextualité consciente, voire délibérée, aux récits de migrations, que ces dernières soient intra- ou intercontinentales. Dans un cas comme dans l'autre, instaurant, dans le récit, la stase qu'impose la découverte, ce type de scène montre un personnage soudain investi par la force invasive d'un présent absolument neuf : climat météorologique, structure urbaine, aspect des rues et des bâtiments, allure et abord des passants. Bien que le topos puisse ponctuellement s'exprimer, comme on l'a vu, à travers une autre ville de l'Ouest africain (comme Conakry chez Camara), Dakar, dont on rappellera qu'avant d'être celle de l'État sénégalais, la cité a été, entre 1902 et 1958, la dernière capitale de l'Afrique occidentale française, apparaît comme l'un de ses référents privilégiés. Ainsi, dans cette littérature, on peut observer qu'à l'aune du sous-continent, et en guise de prélude lorsque les itinéraires se poursuivent au-delà des mers, l'arrivée puis le séjour à Dakar exercent un rôle catalyseur dans les parcours des personnages et des narrateurs, analogue à celui que pourra produire la rencontre avec Paris. L'émerveillement initial déclenche une phase d'individuation, en l'occurrence constitutive d'un parcours migratoire supposant un détachement du groupe, puis celle-ci, grâce à la mise en réseau favorisée par la grande ville, permet dialectiquement l'actualisation de solidarités nouvelles (Garnier 2012 : 110). À cet égard, Dakar n'apparaît pas comme une cité relevant de la périphérie de l'Empire, mais bel et bien comme un lieu capital, où se renouvèlent et se redistribuent les significations aimantant les parcours biographiques des personnages.

Aussi peut-on comprendre pourquoi la ville, qu'elle forme ou non la première étape d'un parcours migratoire à poursuivre au Nord, que ce soit en tant que capitale nationale, ou en tant que pôle sous-régional, fait de longue date l'objet de ces scènes quasiment épiphaniques, où un sujet vient à la rencontre de lui-même, dans les possibles qu'il y discerne. De manière plus itérative, Climbié, le personnage éponyme du récit autobiographique de Bernard Dadié (2002 [1953] : 123), advient à lui-même, comme sujet de l'émancipation anticoloniale, lors de ses pérégrinations dans ce qui est alors la tête de l'AOF, une ville qu' « il a dans la peau », et dont il éprouve, lorsqu'il s'en arrache, la puissance de l'empreinte qu'elle a inscrite en lui. $\mathrm{Au}$ milieu du $\mathrm{Xx}^{\mathrm{e}}$ siècle, ce type d'expérience dakaroise n'est pas propre au sujet africain colonisé : c'est de sa « rencontre » avec des villes africaines, Dakar en particulier (en 1946 puis 1954), que G. Balandier (1957 : 231) fait procéder l' «exploration nouvelle » à laquelle il s'est livré ensuite. 
M. Leiris, lui, espérant fraterniser dans la liesse suscitée par la chute de Berlin à l'issue de la Seconde Guerre mondiale, recherchant «l'effervescence », est finalement brutalement décillé quant à la possibilité pour Dakar, «ville noire », d'être « un paradis terrestre et non un port comme tous les autres ports avec sa pègre prête aux mauvais coups » (Leiris 1955: 171). La découverte peut ainsi, dès cette période précédant les Indépendances, suivant les acteurs et les contextes, osciller entre euphorie et dysphorie. Cette seconde inflexion, anecdotique ou rare jusqu'aux années 1960, va cependant prendre par la suite une place de plus en plus significative dans les représentations offertes de la capitale sénégalaise. Le portrait de la cité se charge de connotations dépréciatives très présentes, notamment pour les parcours allant de la campagne à la ville ${ }^{1}$, dans le « roman de la désillusion » qui s'écrit à partir de 1970, et persiste çà et là à s'actualiser localement, jusque dans des productions récentes (Samb 2008 ; Gueye 2011).

Ces évolutions dans la représentation littéraire de la ville viennent souligner combien il est malaisé de disjoindre l'expérience qui en est faite, et l'image qui en est alors donnée : chaque expérience vécue à Dakar, puis racontée, s'offre aussi comme une lecture de la ville, et vient nourrir les lectures postérieures. Chez Socé et Sadji, la capitale ouest-africaine puise, dans son dynamisme propre, les aliments du rôle catalyseur joué dans la destinée des personnages. Plus tard, c'est dans la recherche d'un tel dynamisme que les parcours s'aimantent vers Dakar, rencontrant de plus en plus fréquemment déception et perdition. Entre euphorie et dysphorie, les expériences nouvelles des migrants qui vont se diriger vers la cité, y passer, ou la quitter pour y revenir, dès lors qu'elles sont retracées dans un récit littéraire, vont s'écrire sur fond de cette production et de cette imagerie antérieure, ambivalente et évolutive.

\section{Fictions contemporaines sous influence}

Les moments de révélation produits par l'arrivée dans la grande ville mettent en balance, faisant appel à un intertexte dense de stéréotypes, des séries sémantiques opposées. À Dakar s'articulent des chemins migratoires et des parcours de vie, mais aussi des entrelacs de discours, des ensembles de représentations qui procèdent au moins autant de dépôts de culture accumulés que d'expériences nouvelles qu'il s'agirait de transcrire. Des extraits d'œuvres aujourd'hui considérées comme des classiques de la littérature

1. Notons que dans le contexte très différent d'un archipel sous le joug portugais, et dont l'extraversion procède de la misère et de la famine endémiques qui y règnent alors, Dakar, dans le roman du Capverdien Baltasar LOPES (1990 [1936]), Chiquinho, fait l'objet d'un investissement ambivalent, tantôt lieu de perdition pour une jeune femme dépressive qui s'y enfonce dans la «débauche » de ses cabarets (ibid. : 151), tantôt refuge où s'enfuir avec «la force d'aimer et de vivre » (ibid. : 229). 
africaine de langue française ont été cités en préambule, car ces titres, parus vers le milieu du $\mathrm{XX}^{\mathrm{e}}$ siècle, appartiennent au canon scolaire, et ce sont notamment ces scènes particulières qui constituent, depuis maintenant des décennies, des morceaux choisis, repris dans maints manuels scolaires en Afrique de l'Ouest, fréquemment polycopiés, et ainsi lus, étudiés et mémorisés en classe par des générations d'élèves. De telles descriptions ont de la sorte contribué à mettre en circulation, puis à diffuser largement des représentations participant de l'imaginaire migratoire relatif à la sous-région tel qu'on peut l'estimer approprié, au moins par une proportion significative de ceux des scolarisés en langue française qui ont poursuivi leurs études jusqu'au niveau du brevet des collèges ${ }^{2}$. Cette littérature scolaire (à la fois diffusée et mise en forme par l'École), formée, plus que par des livres, par des lectures itératives de fragments exemplaires aux yeux des éducateurs, produit des agrégats d'images et d'énoncés, constituant une part de la toile de fond d'une production littéraire ultérieure et endogène. Il s'agira de se demander ici dans quelle mesure de telles représentations ont encore alimenté cette dernière, et si celle-ci, à travers les perceptions qu'elle dévoile, les enchâssements et branchements narratifs qu'elle met en œuvre, notamment s'agissant de ce lieu nodal que forme, encore aujourd'hui, la capitale sénégalaise, réserve en la matière ou non un sort particulier aux mouvements migratoires Sud-Sud, lorsqu'elle prend ces derniers pour objet.

On s'appuiera à présent sur un corpus contemporain de quatre productions littéraires (fictions ou textes autobiographiques), dans un empan d'une douzaine d'années, et depuis la périphérie du champ littéraire francophone ${ }^{3}$. Toutes les quatre ont pour objet commun un parcours migratoire relatif à l'Afrique de l'Ouest, qu'il demeure circonscrit à cette dernière (dans deux cas) ou qu'il déborde jusqu'en Afrique centrale (en une occasion) ou au Maghreb et en Afrique australe (en une autre). Dans l'ordre chronologique de parution, la première relève de ce que l'on appelle parfois la paralittérature puisqu'il s'agit d'un album de bande dessinée, Farafina Express (Saleh et al. 1998), dont les dessins ont été réalisés par El Hadj Sidy Ndiaye et le scénario co-écrit par Karine Saleh, Karim Dahou et Yann Nachtman,

2. Les extraits cités ont généralement pris place dans des manuels destinés aux classes de $5^{\mathrm{e}}$ et de $4^{\mathrm{e}}$ (élèves de douze à quatorze ans environ).

3. Rappelons que la sociologie du champ littéraire, ayant mis en évidence l'exceptionnelle centralisation du champ littéraire francophone, a aussi souligné, à partir de la formulation du couple centre/périphérie, qu'une littérature ou un ensemble littéraire "périphérique » peut se comprendre comme un sous-système moins prestigieux, dans une relation de dominé à dominant vis-à-vis du centre prescripteur et dispensateur de légitimation. En ce sens, le corpus abordé peut être qualifié de «périphérique » à trois égards : d'un point de vue géographique, éditorial, et parce que certaines œuvres relèvent plutôt de ce qu'on appelle parfois «paralittérature ». Il n'est évidemment pas question ici d'un quelconque classement ou jugement de valeur, et l'on peut également ajouter qu'un champ littéraire se trouve justement soumis, à travers les stratégies déployées par ses acteurs, à des dynamiques d'évolution et de redistribution des positions. 
appuyés par un comité de lecture formé de personnalités du monde associatif. L'album a été publié par l'organisation non gouvernementale ENDA TiersMonde basée à Dakar, avec le concours du Comité international des femmes africaines pour le développement (CIFAD), de la Fédération internationale des professeurs de français (FIPF) et le soutien financier de l'Agence de la Francophonie, dans le cadre d'un projet intitulé « 2000 autrement». On perçoit ainsi d'évidence que les buts poursuivis auprès du jeune public, cible de la publication, ne sont pas exclusivement récréatifs et consistent plutôt à véhiculer de concert un ensemble de messages, de contenus didactiques précis (sur la santé, la récupération des déchets, la confection de foyers améliorés, les feux de brousse, etc.) et des représentations d'une " Afrique vive et dynamique $»^{4}$, alternative aux clichés afro-pessimistes.

La deuxième production, D'Abidjan à Tunis (Ndoye 2007), est un texte autobiographique qu'on doit à une romancière sénégalaise dotée d'une importante notoriété (restée plutôt circonscrite au champ littéraire ouest-africain), Mariama Ndoye. Cette dernière a longtemps résidé en Côte-d'Ivoire, avant de devoir en partir sous la pression des événements politiques. La troisième, Le Regard de l'aveugle (Samb 2008), est un roman à l'esthétique réaliste voire naturaliste, dont l'auteur, Mamadou Samb, émane du même milieu associatif dakarois que celui qui a produit Farafina Express [FE]. La quatrième enfin, bien que francophone, se distingue des précédentes par une publication aux États-Unis. Cependant, la maison d'édition qui la publie constituerait, m'a-t-il été indiqué dans la librairie dakaroise ${ }^{5}$ où je l'ai achetée et qui proposait plusieurs ouvrages de cette maison, une entreprise de la diaspora sénégalaise. Destinée, sous-titré Le récit personnel d'un voyage forcé, récit autobiographique d'Abdoulaye Yansané (2010) dit « Boy Colobane », a pour cadre trois pays d'Afrique de l'Ouest (Guinée, Mali, Sénégal). Une grande partie de l'action se déroule dans la capitale sénégalaise et plus précisément dans Colobane, le quartier populaire marqué par les flux multiples de populations venues d'ailleurs.

Deux de ces récits (FE et Le Regard de l'aveugle [RA]) émanent donc du milieu associatif œuvrant pour le développement, et poursuivent des visées didactiques, en prétendant influer sur le comportement des acteurs (jeunes adultes, grands adolescents) à travers des fictions narratives. Farafina Express reprend, sous forme de bande dessinée, le schéma du roman d'éducation mâtiné de récit d'aventures. Il relate le parcours circulaire en Afrique, du Sénégal au Congo RDC et retour, d'un jeune homme encore naïf et peu assuré, Babacar Bah dit Bab's, engagé dans ce périple ${ }^{6}$ avec la mission de ramener au pays Maguette Fall dit Max, un aîné expatrié depuis près de vingt ans,

4. Texte figurant sur la deuxième de couverture.

5. Librairie Athena (33, rue Jules-Ferry, Dakar-Plateau).

6. Celui-ci lui a été imposé par la nécessité de fuir son quartier sous la vindicte d'un chef de famille traditionnel, l'accusant — à tort — d'avoir engrossé sa fille. 
dont les idéaux révolutionnaires se sont brisés au fil de douloureuses expériences. Le Regard de l'aveugle, de facture plus classique, relate le parcours migratoire riche, malgré sa linéarité, d'une jeune Malienne, depuis son village ravagé par l'onchocercose jusqu'à Dakar en passant par Bamako. Offrant un tableau diversifié des différentes formes de migrations et des milieux sociaux dans la capitale sénégalaise, il produit et relaie un discours dense sur les migrations juvéniles, tant dans le cadre sous-régional que dans le cadre national. L'aventure tragique de la jeune héroïne se veut exemplaire et dissuasive, tant dans son ensemble que dans le détail de l'itinéraire et des rencontres. Il faut en outre noter que le livre a fait l'objet d'une appropriation significative par un lectorat jeune, puisqu'il a remporté, en étant «plébiscité » dans ce cadre, selon plusieurs professeurs de français partie prenante de l'organisation, un prix littéraire décerné en 2010, à une œuvre éditée localement, par un jury national de lycéens au Sénégal. L'ambition portée par le récit de toucher des lecteurs adolescents et d'influencer leurs représentations, voire leurs comportements, semble à cet égard avoir été au moins partiellement satisfaite.

Les deux autres productions (D'Abidjan à Tunis et Destinée) présentent un fort substrat autobiographique, sans s'inscrire pour autant dans un cadre générique étroitement défini. D'Abidjan à Tunis [AT], auto-édité ${ }^{7}$, procède de la spéculation autobiographique d'une Sénégalaise, appartenant au milieu des cadres de haut niveau d'une organisation bancaire interafricaine, qui, en raison des troubles politiques, a dû quitter Abidjan où elle résidait depuis de longues années pour se réinventer une vie à Tunis (où s'est repliée la Banque africaine de développement) : «D'Abidjan à Tunis en passant par Dakar ou Paris, au gré des pérégrinations d'une famille diplomatique, les tableaux de vie se bousculent et s'entrechoquent dans mon esprit, débordants de messages divers » ( $A T: 9)$. La période de référence est la première décennie du $\mathrm{XXI}^{\mathrm{e}}$ siècle, mais la remémoration est au principe de nombreux retours en arrière, d'amplitude temporelle variable, qui focalisent en outre la narration sur différents espaces situés au Sénégal. Enfin, Destinée [De], dont l'auteur réside désormais aux États-Unis, est un récit autobiographique relatant une migration familiale de la Guinée au Sénégal à l'époque de la dictature de Sékou Touré, et dépeignant aussi bien des réseaux migratoires familiaux à travers la sous-région (entre Guinée, Mali et Sénégal) qu'à Colobane, durant les années 1970 et 1980.

Ces œuvres sont donc diverses par le genre, par les matériaux narratifs convoqués, empruntant à des moments historiques, des lieux, des segments

7. Les autres œuvres de l'auteure ont été publiées soit à Paris chez Présence Africaine (pour la première), soit à Dakar, auprès des Nouvelles Éditions Africaines, et parfois, cas le plus fréquent, à Abidjan, auprès du CEDA, des Nouvelles Éditions Ivoiriennes, ou des éditions Éburnie : voir Lire les femmes écrivains et les littératures africaines, "Mariama Ndoye Mbengue », <http://aflit.arts.uwa.edu.au/ NdoyeMariama.html>, consulté le 14 avril 2012. 
de la société variés et parfois aux deux extrémités du prisme social (dans $R A$ et $A T$ par exemple, les deux personnages féminins principaux sont dans un cas une très jeune mendiante, dans l'autre une femme d'âge mûr, appartenant au milieu de la haute fonction publique), par les visées poursuivies. Deux d'entre elles ( $F E$ et $R A$ ) ont été publiées et distribuées par des réseaux militants d'activistes du développement. L'une des deux ( $F E$ ), qui est d'ailleurs une œuvre collective ayant fait l'objet de plusieurs étapes de validation, a même bénéficié pour cela d'un soutien institutionnel significatif. La visée idéologique, la volonté d'influer sur le devenir de la société y sont donc premières. Pour les deux autres (De et $A T$ ), parce qu'elles relèvent, avec des nuances, de l'autobiographie, cette visée et cette volonté sont moins manifestes, ce qui ne signifie pas pour autant qu'elles soient absentes. Centrées sur les voyages et les exils de sujets singuliers, elles n'en visent pas moins, chacune, à fournir des modèles d'intelligibilité aux destinées collectives, dans la relation qu'entretiennent les parcours individuels avec l'histoire politique et sociale de l'Afrique de l'Ouest.

Le choix d'un tel corpus vise à éclairer les perceptions suscitées par les migrations Sud-Sud, dans le contexte-même où ces œuvres ont pris naissance. En effet, celles-ci sont d'autant plus à même d'informer sur ce qu'il en est actuellement de la circulation des représentations et de la configuration des imaginaires, qu'elles ont été éditées récemment et diffusées dans la sous-région par de petites structures locales. Ainsi orientées vers un public de proximité (ou, dans un cas, diasporisé, mais bel et bien segmenté et singularisé en tant que tel), elles ne procèdent pas a priori de l'extraversion analysée par différents chercheurs (Halen 2001 ; Paravy 2001) dans d'autres productions qui font habituellement l'objet de l'attention de la critique littéraire internationale, et qui visent le centre du champ littéraire, ainsi que par surcroît la reconnaissance d'un public et d'un marché européens, ou plus largement «occidentaux ». Or cette extraversion amène fréquemment soit à devancer les attentes supposées d'un tel lectorat, en reconduisant celles des représentations stéréotypées, assignant aisément l'Autre à un pur exotisme, qui circulent de façon privilégiée au niveau global, depuis le Nord et ses schèmes d'appréhension du Sud, soit, dans une perspective de critique et de contestation, à tenter de déconstruire de telles représentations, entre autres à travers la promotion de figurations alternatives. Même en ce cas, le Nord, avec l'agencement des représentations qu'induisent les perceptions dont il est l'origine au niveau global, demeure alors la principale référence. On ne postule évidemment pas pour autant une stricte compartimentation des imaginaires, localement référés. Mais, dans la mesure où l'on s'intéresse d'abord à la circulation des représentations à un niveau local élargi (le souscontinent), ce sont ces dernières que l'on cherchera d'abord à établir, quant aux trajectoires migratoires, quant à la ville comme nœud de virtualités dans les parcours biographiques, pour en revenir ensuite à la confrontation avec celles qui circulent au niveau global. 


\section{Trajectoires migratoires et parcours biographiques}

Les déplacements des personnages de ces quatre récits, tout comme les formes prises par leur narration, illustrent des types de trajectoires différents. Le mouvement migratoire, linéaire ou discontinu, détermine l'élan ou la déchéance dont bénéficie ou souffre le personnage dans son cheminement biographique. Deux de ces trajectoires, l'une ascendante (De), l'autre descendante $(R A)$, sont plutôt vectorielles ; une autre est circulaire $(F E)$, la quatrième relativement erratique et brisée, ce qui se traduit également dans la construction temporelle de l'ouvrage (AT). L'observation de la couverture de Destinée fournit d'emblée plusieurs indications, iconiques et textuelles, sur l'inscription du parcours raconté — « entre la Guinée de Sékou Touré et [le] Sénégal d'adoption » - dans un cheminement biographique au plus long cours. Commencé dans l'exil, ce parcours est dynamisé à Dakar, grâce à des études « au prestigieux lycée Blaise Diagne » et à l'obtention du baccalauréat, puis consacré par la préparation d'un $P h D$ et d'un poste d'assistant professeur à l'Université du Tennessee. Le parcours du personnage principal, un jeune garçon, prend ainsi doublement sens : il se présente comme une réécriture contemporaine, tournée vers l'Amérique, de L'Enfant noir ; il s'héroïse en success story à travers la mise en exergue du statut social conquis par l'auteur. Inauguré dans les affres suscitées par les menaces que fait peser le régime dictatorial de Sékou Touré sur la famille Camara (le père a rencontré la mère du jeune Amidou «lors d'un de ses déplacements diplomatiques à Dakar»[De: 16]), puis les difficultés et les incertitudes de l'exfiltration du personnage principal et de son frère hors de Guinée, ce parcours se stabilise et se linéarise grâce au courage de la grand-mère sénégalaise, ainsi qu'au long séjour à Dakar du héros qui y grandit sous la protection de son aïeule, et peut finalement envisager de mettre le «cap sur l'Amérique » (titre du dernier chapitre). Cette destination donne précisément à l'aventure, jusqu'alors incertaine, le sens d'une «destinée », démontrant, même si « la lutte de l'être n'est jamais finie », que «tous ceux qui errent ne sont pas des êtres en perdition » (De : 127).

Initialement, le parcours de la narratrice du Regard de l'aveugle est aussi ascendant, son père réussissant « la prouesse de [1']envoyer à Bamako pour entrer à l'école française », et faisant d'elle la première fille de sa famille à « se rendre loin en ville pour suivre des études » $(R A: 28)$. Mais, de retour dans son village d'origine décimé par l'onchocercose, elle y découvre son père aveugle et le départ s'impose. Elle mendie d'abord à Bamako afin d'assurer une subsistance à tous deux, mais la concurrence régnant entre les mendiants pousse père et fille à prendre la décision d'un nouveau départ, pour Dakar, une « obsession», un «point de mire» $(R A: 111)$. Si « l'exode finit par s'imposer» (ibid. : 123), les «voyageurs clandestins » qui vont «à l'aventure » (ibid. : 119) mettent de longues semaines, faisant étape, le long des rails, à Tambacounda puis à Kaolack, à parvenir au but de leurs « rêves » (ibid. : 129). Ouly, cruellement déçue par Dakar, y devient prostituée et 
toxicomane. La capitale sous-régionale aura été, pour elle comme pour d'autres, un lieu de perdition et de déchéance, comme l'annoncent l'incipit et la structure du roman, puisque la jeune femme, malade du sida, témoigne depuis son lit d'hôpital, et que le deuxième chapitre s'ouvre sur une analepse, la ramenant vingt ans plus tôt, dans son village situé au nord de Bamako. Partout étrangère dès le moment où elle s'éloigne de son milieu d'origine, Ouly, à la fin de son récit, émet le souhait d'un ultime retour, dans l' « humble cimetière » (ibid. : 264) de son village natal. Alors que le départ de Guinée, grâce à l'entregent de la grand-mère sénégalaise, s'avère en tout point salvateur pour le jeune Amidou, Ouly, elle, parce qu'elle est une fille, ne peut nulle part trouver sa place, ni là où elle est née, ni là où la portent ses pas. C'est ce qu'illustre l'achèvement de la boucle géographique et biographique accomplie par le roman.

Celle que réalise le personnage principal de Farafina Express possède une tout autre signification, puisque le jeune homme, ayant précipitamment quitté Dakar afin d'échapper au courroux d'un voisin, ayant rencontré heurs et mésaventures tout au long de sa route, s'en retourne dans son quartier plein d'usage et raison, prêt à entreprendre, mettant ainsi fin à l'indécision pusillanime et nonchalante de sa prime jeunesse. L'itinéraire circulaire du jeune «aventurier» $(F E: 88)$ le mène de Dakar à Bamako et Ouagadougou, puis Douala et Kinshasa avant un retour par Abidjan, et lui fait croiser toutes sortes de personnages, malfaisants ou secourables, figurant différents visages d'une Afrique plurielle, ancrée dans le passé (le père outragé), intemporelle (la jeteuse de cauris, le cultivateur...) ou branchée sur la modernité (les rappeurs, l'administrateur de site Internet, la styliste à succès, ainsi que quelques malfrats de diverses envergures). Le récit prend en outre en compte, sous forme de tiroirs enchâssés, les deux périples moins détaillés de personnages secondaires, celui, contemporain, qui mène la jeune Oumou à Abidjan et celui, antérieur, de Max lui-même. L'un, celui de la jeune femme, couturière de talent, est un parcours de réussite entrepreneuriale ; l'autre, celui du « grand frère » qui, à Kinshasa, a sombré dans l'alcoolisme et la misère, est voué à l'échec et à la déchéance. Toutefois, l'aîné est sauvé par l'intervention du cadet, qui parvient à le ramener chez lui à Dakar, au sein des solidarités agissantes du quartier. De multiples hypotextes ${ }^{8}$ peuvent être convoqués pour ce récit tout à la fois scolaire, ludique et didactique, jusqu'au conte philosophique et à Candide (Voltaire 1949 [1775]), la morale finale de l'histoire pouvant être ramenée à un «Il faut cultiver notre jardin » (ibid. : 218) renouvelé : "s'investir dans des activités utiles au quartier» ( $F E$ : 84) plutôt que s'échiner — en vain et au risque du désespoir, comme Max — à changer le monde. Le récit d'aventures vise ainsi à éduquer le lecteur à travers contre-modèles et modèles ; il est aussi récit d'éducation

8. Gérard GenetTe (1982: 13) définit l'hypertextualité comme «toute relation unissant un texte B » (l'hypertexte) "à un texte antérieur A » (l'hypotexte), "sur lequel il se greffe d'une manière qui n'est pas celle du commentaire». 
au sens où l'aventure migratoire, avec les risques qu'elle impose, «permet à l'homme » encore en devenir qu'est le jeune Bab's «d'advenir et de s'aguerrir » (Bredeloup $2008: 281)$.

En Afrique comme ailleurs, en littérature comme en sciences humaines, il a souvent été rappelé qu' «aventure rime avec jeunesse » (ibid. : 298), parce qu'elle comporte une dimension initiatique, parce que l'intensité de la vie vécue qu'elle révèle est indissociable de prises de risques souvent multiples. À cet égard, le récit des aventures migratoires de Mariama Ndoye fait exception, puisqu'il met principalement en scène une grand-mère (il est d'ailleurs dédicacé à la "génération [des] petits-enfants » de cette dernière). Deux autres personnages, plus jeunes, et tous deux décédés au moment de l'écriture, y tiennent également une place significative : il s'agit de deux domestiques, une jeune bonne sénégalaise originaire de Casamance, Mouskéba, disparue lors du naufrage du Joola ${ }^{9}$, alors qu'elle revenait d'un séjour de trois ans en Côte-d'Ivoire, auprès de la famille de la narratrice ; et Billy, le boy d'Abidjan, dont on comprendra par la suite qu'il a succombé au sida, et à qui la narratrice («Madame va te raconter» [AT : 9]) entreprend de relater les «pérégrinations » de la famille, entre Abidjan où ont été vécues des années d'insouciance, Tunis où elle «apprend cahin-caha à [s']adapter à un nouveau milieu » (ibid. : 14), Dakar d'où remontent, avec ceux d'escales plus récentes, les souvenirs enchantés d'une enfance choyée, le Djoloff découvert d'un oil neuf lors de vacances au pays (ibid. : 28), et même un court séjour en France, lors duquel elle expérimente l'extension des « phobies du citoyen français moyen » à l'encontre des étrangers (ibid. : 35).

Si les personnages de Mouskéba et Billy sont emblématiques d'une condition ancillaire corrélée à une mobilité campagne/ville, ils sont aussi représentatifs de l'élargissement international, du moins dans le cadre ouestafricain, de celle-ci : Billy est originaire du Burkina et, quand il apprend sa maladie, envisage de partir se faire soigner au Ghana; le service de la famille a conduit Mouskéba, après un premier trajet de la Casamance à Dakar, jusqu'à Abidjan. Quant à la narratrice, elle approche sa propre mobilité, non circonscrite à l'Afrique de l'Ouest même si elle y a trouvé son premier espace de déploiement, à travers deux paradigmes antagoniques (ce qui peut aussi contribuer à l'explication du caractère brisé de la narration) : l'exil et le cosmopolitisme. L'exil est appréhendé à la fois comme la rupture des habitudes («Les malheurs de l'exil neuf, pas de domestique à plein temps, de jour comme de nuit, comme à Abidjan et Dakar » [AT: 80]), la nostalgie de «Baabi », la ville, désormais en proie à la guerre, où les enfants ont grandi (aussi les « exilés » sont-ils «ivoiriens de naissance ou de cœur » [ibid. : 78]), et la condition d'étrangère à Tunis, une ville « frileuse en hiver» (ibid. : 9), dans un pays dont on ignore la langue et méconnaît certains

9. Rappelons que ce bateau surchargé, qui effectuait la liaison entre la Casamance et la capitale du Sénégal, sombra le 26 septembre 2002, entraînant la mort de près de deux milliers de personnes et suscitant un traumatisme national. 
usages, où l'on est parfois la cible de commentaires malveillants, mais aussi l'objet d'élans de solidarité. Mais un ethos cosmopolite découlant du statut social privilégié de la narratrice («Madame et ta deuxième famille sillonnent les aéroports du monde » [ibid. : 148]), associé à une condition diasporique consubstantielle à l'identité léboue dakaroise, prend finalement le pas sur ces misères, somme toute limitées, de l'exil vécu :

« Notre peuple serait passé par le Fezzan et le Darfour. La branche des Guermantes serait restée au Tchad dont les Toubbous seraient des Lébous obstinés "lebu yu lubbu" qui ont choisi de ne pas poursuivre la route. Une fois au Sénégal, il y eut deux vagues de migrations, l'une vers Jelekk, Yoff, l'autre vers Jalaw, Yène, Toubab Dialaw. [...] C'est notre facilité à accepter l'autre, à le mettre à l'aise en nous effaçant presque qui a fait dire à une animatrice [...] que Dakar kenn dekku fi, personne n'est originaire de Dakar. Sa déclaration péremptoire nous a fait avaler de travers, nous tous Lébous de la diaspora, à l'heure du dîner. "Et si on la ramenait dans sa brousse celle-là ?" nous sommes-nous tous dit, de New York à Djeddah » (ibid. : 100-101).

Aussi les dernières pages du récit voient-elles la narratrice découvrir «Jobourg », avec «cette autre Afrique si symbolique pour nous tous et son peuple aussi coloré que l'est son drapeau» (ibid. : 154), source d'espoir pour «Baabi » (Abidjan) et la Côte-d'Ivoire. La composition en mosaïque dispersée du livre correspond ainsi à la multiplicité des trajectoires accomplies tant par la narratrice et des membres de sa famille que par ses deux domestiques. Mais elle répond également à la quête lancinante de la mémoire, alimentée par la nostalgie du pays quitté («qu'y a-t-il de bien réel dans toute cette aura qui subitement entoure ce pays frère » [ibid. : 50]) et la tentation d'effacer les deuils grâce à l'anamnèse : «Et l'esprit d'aller à la chasse des souvenirs quelques années en arrière. Nafy prépare son entrée en $6^{\mathrm{e}}$, Mouskéba n'est pas encore rentrée en Casamance, tout est parfait, nous n'avons pas de bleu au cœur, elle n'a jamais pris ce maudit bateau... » (ibid.).

Entre local et global, la ville, nœud de virtualités

La capitale sénégalaise aimante les trajectoires bien au-delà du seul espace national. En tant qu'espace urbain des possibles, elle permet d'envisager et de rêver leur relance. Comme on l'a vu, au sein de trajectoires circulaires ou hachées, Dakar peut être lieu du retour de l'enfant prodigue (FE) ou du ressourcement $(A T)$; encore celui-ci demeure-t-il entièrement poreux à l'Ailleurs, le peuple lébou parvenant à incarner, en une parfaite symbiose, territorialisation dans la presqu'île et identité diasporique, en amont comme en aval (AT : 100-101). Dans le cadre de trajets vectoriels, le passage par Dakar catalyse l'ascension (De) ou la chute $(R A)$, lorsque pour Ouly, l'arrivée à Dakar, finalement « à l'image des autres capitales africaines », « ville sans 
rêves, sans contes, où l'avenir, le passé et le présent se résument au quotidien dans une quête effrénée du gain » $(R A: 132)$, représente une terrible désillusion : «Un mois après, le Dakar de mes rêves s'était évanoui en moi dans une mare de décrépitudes, de déceptions et de désespérances » (ibid.).

Ainsi, dans les imaginaires mobilisés, la ville est-elle l'objet d'investissements contradictoires : tantôt milieu hostile, dont la représentation charrie les nombreux stéréotypes littéraires attachés à la grande ville et/ou aux basquartiers, comme lieu de perdition et de désillusion pour les ruraux et les étrangers $(R A)$, tantôt havre pour la réinvention de soi, nimbé d'une aura d'habitabilité et d'hospitalité aux exilés ( $A T, F E$, De). La presqu'île est une terre d'accueil immémorial où chacun peut librement s'enraciner, d'où chacun peut à sa guise prendre son envol (AT); certains quartiers précisément dénommés sécrètent de la solidarité (la Médina dans $F E$ ) et de l'urbanité (Colobane dans $D e$ ). Malgré les difficultés d'acclimatation résultant des différences culturelles entre deux pays pourtant frontaliers (De: 67), le petit Amidou peut «s'épanouir» à Colobane, «dans l'insouciance et en totale sécurité » (ibid.), recouvrant « une certaine joie intérieure de vivre » grâce à de « petites bénédictions » pénétrant l'atmosphère autour de lui (ibid. : 84). L'urbanité d'une «vie paisible et calme » à Dakar se concentre dans les week-ends passés à Grand-Place où des « vieillards » jouent à la «belotte » [sic] en formant « un vrai cercle fraternel », et surtout « un lieu d'éducation de la rue » (ibid. : 94-95). En effet, les joueurs de cartes devisent aussi de leurs voyages récents, offrant «une véritable leçon de géographie » au jeune Amidou, qui, de la sorte, parvient aussi à nourrir « une vraie affinité pour les langues, la culture générale et la diversité culturelle », car il entend les gens « converser en anglais aussi bien qu'en wolof, en russe, en portugais et en arabe avec une facilité égale » (ibid. : 96). Cet « afropolitanisme ${ }^{10}$ simplement vécu ouvre à la fois sur le parcours d'excellence dans l'extraversion qui sera celui du jeune homme, et sur une disposition éthique dont les habitués de Grand-Place incarnent le modèle collectif : "Grand-Place apprit à Amidou à douter des stéréotypes infondés mais aussi à apprécier l'importance de l'amitié, de la générosité, de la plaisanterie et du rire » (ibid.).

Dakar, à travers la richesse humaine et la singularité de ses quartiers, apparaît dès lors comme une cosmopolis (Malaquais 2006a), une «villemonde » ou ville-relations :

«Une ville, qui peut être le lieu de tant de souffrances, d'injustices, de malheurs étouffés, de désespoirs sans horizons, devient par là, entrant dans l'imaginaire du monde et complétant cet échange et commettant Relation, le symbole et le vecteur

10. Achille Mrembe (2006) définit l'afropolitanisme comme une «sensibilité culturelle, historique et esthétique » reposant sur la «conscience» de l'«imbrication de l'ici et de l'ailleurs », une "relativisation des racines et des appartenances primaires », une "manière d'embrasser, en toute connaissance de cause, l'étrange, l'étranger et le lointain », une "capacité de reconnaître sa face dans le visage de l'étranger» et de «domestiquer l'in-familier». 
d'espérances nouvelles. [...] Une ville rassemble et signifie la région où elle s'est fondée, mais elle s'ouvre tout autant aux systèmes de relations qui se sont tramés entre les cultures du monde»(Glissant 1997: 250).

Les personnages investissent ainsi, à travers Dakar, un espace urbain porteur, de longue mémoire, d'un imaginaire déployé et ramifié à l'échelle sous-continentale. Ils traversent et revisitent des significations déjà inscrites, à travers littérature, cinéma et discours circulants, dans les quartiers, les rues, les transports de la capitale sénégalaise. La peinture peut viser le réalisme social, ainsi, dans $R A$, de la description de la chambre occupée à la " cité Bissap », " un bidonville constitué d'un amas de baraques, presque pareil aux autres quartiers flottants », «imposé par la force des choses dans un terrain destiné à la culture de l'oseille (Bissap) situé entre les HLM, les maisons modernes de la Sicap et les quartiers populaires de Grand Dakar », une chambre "moins confortable que celle que l'on avait à Yeumbeul », dans laquelle Ouly et son père voisinent avec «plus de quinze jeunes filles sérères qui [sont] venues en ville pour travailler comme bonnes » et «cherchent à recréer l'ambiance de la vie communautaire au village en évoquant les nostalgiques rythmes de leur sol natal» $(R A: 182)^{11}$. Mais le pittoresque pur vient rapidement à la rescousse pour caractériser et singulariser Dakar par rapport à d'autres capitales de la sous-région, avec la mention des « cars rapides » (ibid. : 183). Chez Ndoye, la description de l'autoroute empruntée pour se rendre à Rufisque permet de confronter la réalité actuelle aux souvenirs d'autrefois. Il n'est cependant pas indifférent que l'énumération des différents véhicules de transport en commun circulant sur la presqu'île serve essentiellement à caractériser une modalité d'être de la société sénégalaise tout entière :

«Les cars dits rapides, les Ndiaga ndiaye du nom de leur propriétaire, les dem dikk (ceux qui vont et viennent, en langue locale) rebaptisés par la population insatisfaite de ses performances, demul dikkul (ils ne vont ni ne viennent !), les taxis clandestins dits clandos, créent d'autres files. Mes compatriotes ont l'imagination fertile. Ils ont qualifié de "mélangeurs" les coûteux échangeurs destinés à décongestionner la circulation routière » $(A T: 94-95)$.

Dakar n'est pas seulement une cité investie par un imaginaire bifide, dysphorique dans $R A^{12}$, généralement euphorique dans les autres récits. Dakar y apparaît aussi comme plus qu'une ville, et autre chose qu'une ville : l'un de ces «mélangeurs » où se jouent intrications et interactions entre un local élargi à un hinterland aussi vaste que la sous-région continentale, et le global où se redéploient les identités diasporiques ( $D e$, $A T$, et dans une moindre mesure $F E$ ). La capitale ouest-africaine des passages fournit un paradigme

11. L'association ENDA-Tiers-Monde, auprès de laquelle l'auteur du roman a travaillé, intervient notamment dans les bidonvilles et quartiers flottants dakarois.

12. Comme dans de nombreux autres romans sénégalais publiés depuis la fin des années 1970. 
comme forme-sens de ce type de nœuds spatio-symboliques que sont les cosmopolis africaines. Il n'est donc pas étonnant que, comme on l'a vu dans la première partie, le fonctionnement du sème dakarois ait été analogue, dans une certaine littérature, à celui du sème parisien : l'horizon migratoire formé dans un premier temps par la cité de la presqu'île du Cap-Vert se convertit bientôt en espace de redistribution des virtualités, tournées vers l'ailleurs, que portent en germe les trajectoires migrantes.

De ce point de vue, les choses ont changé depuis le milieu du $\mathrm{XX}^{\mathrm{e}}$ siècle : à cette époque, arriver à Dakar garantit un choc ou un éblouissement analogues à ceux qu'on éprouve en arrivant à Paris, alors capitale de l'empire colonial. De nos jours, la trajectoire européenne ne forme que l'une, parmi d'autres, des pistes empruntées par les destinées migrantes. Dans RA, c'est Sidiki, un déplacé par la construction d'un grand barrage hydro-électrique, qui, rencontré à Goudiry, cherche à « rassembler assez d'argent avant d'aller à Dakar, et de là, trouver un moyen par bateau ou par avion » (RA : 124). Ouly le retrouve plus tard, balayeur dans l'hôpital où elle est internée : comme les siens, les rêves du jeune homme se sont "brisés sur les rives de Dakar» (ibid. : 256). Le roman ne va pas au-delà de l'articulation factuelle, ici impossible, entre migration intra-africaine et hors d'Afrique. Dans $F E$, le «bon voyage » qui «forme la jeunesse » et mène jusqu'à Kinshasa à la découverte de la richesse multiple du continent, un garçon qui n'a jusqu'alors « jamais dépassé Rufisque » (FE : 10) est implicitement présenté comme une alternative aux mirages européens ${ }^{13}$, et l'extraversion supplantée par des modèles endogènes : mode africaine réalisée à partir de tissus locaux, studios d'enregistrement pour les rappeurs, etc. Parti de Dakar avec l'express Dakar-Bamako, Bab's y revient en avion, survolant le Plateau et Gorée qu'il « n'espérait plus revoir» (ibid. : 81), et c'est l'association qu'il fonde avec ses amis qui porte le nom de Farafina Express. À travers la réussite de son périple et les réalisations multiples qui se profilent à la suite, la Médina, rompant le lien de dépendance avec le quartier des affaires du Plateau (ibid. : 85), branche directement Dakar sur le reste de l'Afrique, dont Bab's a appris à connaître une bonne partie, et ses réussites, préludes d'un nouveau siècle. C'est donc cette Afrique-là qui est présente à Dakar, non seulement à travers ses ressortissants (l'amie camerounaise de Bab's, par exemple) et ses voyageurs (Max), mais grâce à la circulation des informations et des représentations, au partage des expériences. Quant aux deux récits autobiographiques, ils franchissent chacun un pas supplémentaire, en permettant de lire, dans Dakar, le creuset d'un être-au-monde afropolitain, où s'articulent des imaginaires élaborés en des migrations centripètes (de Conakry à Colobane, de la Casamance au Plateau), et ceux qui préfigurent des mobilités centrifuges à travers des identités diasporiques.

13. De plus, à l'orée des années 2000, la question posée par ces derniers est moins brûlante qu'elle ne l'est une douzaine d'années plus tard. 
Motifs imaginaires, enjeux existentiels

Cette mise en récit d'événements de vie que détermine et ordonne la mobilité se déploie en outre sur un fond mémoriel associant, à la scansion de l'Histoire collective, des motifs insistants. Ceux-ci sont non seulement communs aux récits de migration Sud-Sud et Sud-Nord, mais entrent également en résonance avec des motifs allégoriques, voire mythologiques, que réactive nécessairement la narration de parcours migratoires, quand des vies risquées se confrontent à des passages obligés : moyens de transport plus ou moins sûrs, franchissement de la frontière, parfois clandestin et périlleux, arrivée en des lieux et des milieux totalement inconnus, rencontre, quête de moyens de subsistance, etc. On se limitera ici à deux de ces topoï, tragédie nationale dans un cas, événement biographique dans l'autre : le naufrage du Joola (AT), le passage de la frontière (De).

Partir, c'est mourir à soi-même pour renaître ailleurs, et autrement, ce que représente allégoriquement le franchissement de l'eau (Diome 2003 ; Gueye 2011 ; Coutinho et al. 2012 ; Mazauric 2012). Mais le symbole peut se retourner, provoquant le scandale et l'horreur, d'autant plus grands que le voyage est un retour. C'est ce qui survient avec le naufrage du Joola : «Comment ce bateau peut-il être mauvais ? Lui qui ramène les élèves à leur école chérie, les étudiants à leurs coquets pavillons de Sanaar et Fann, les vendeuses du port à leurs étals providentiels ?» (AT: 5). Le souvenir épouvantable scande le récit de Ndoye, il l'inaugure en une narration antérieure : dans les premières pages du livre, une pythie casamançaise au «fleuve de paroles, de menaces, de suppliques, d'invectives » de qui personne n'accorde attention prophétise, de la plage où elle erre en vain à portée du navire en train d'être chargé, la «nuit satanique» (ibid. : 5-7). Celle-ci est en même temps directement corrélée aux «tares » qui ont alors «pris le dessus » au Sénégal (ibid. : 7). Les dimensions mythologique et socio-historique de l'événement sont ainsi d'emblée mêlées : "Le Joola comme un baobab séculaire s'est couché et sous lui nos cœurs de Sénégalais ont chaviré dans l'eau noire, âcre et mugissante »(ibid.). Ouvert sur ce traumatisme national touchant à la noria, qu'il a un temps rompue, entre Casamance et capitale, le livre se clôt sur ses suites, en lien avec l'émigration: la narratrice évoque d'abord, à l'intention de Mouskéba, qui a péri dans le naufrage, les veuves qui «travaillent maintenant en Espagne pour nourrir leurs enfants », avant d'en venir à « nos fils et nos frères » ayant « embarqué dans des pirogues trop lourdement chargées qui réveillent en nous d'affreuses images » (ibid. : 158-159), une tragédie derechef collective réactivant le souvenir d'une autre.

Dans Destinée, alors qu'à Conakry, Zéma Camara a été conduit en détention par des militaires, sa jeune épouse, d'origine sénégalaise, décide d'envoyer ses deux très jeunes fils chez sa mère à Dakar. Maam Matty a conseillé d'amener les enfants dans un village frontalier du Mali, où elle les attendra, avant de rejoindre avec eux le Sénégal en train (De : 24). 
L'aventure est «périlleuse », il s'agit d'une «solution risquée exigeant un long voyage à travers la jungle touffue »(ibid.), mais les deux femmes estiment n'avoir pas d'autre choix. La jeune mère bénéficie toutefois de la complicité d'un officier, redevable à son mari, qui lui propose d'organiser la fuite des enfants. La première tentative échoue. Lors de la seconde, la «mission» de l'officier secourable est «accomplie », comme le titre du chapitre en informe par avance le lecteur. L'obstacle sur lequel avait buté la tentative précédente est cette fois franchi : il s'agit d'un «pont rudimentaire qui vous foutait la trouille jusqu'à la moelle, même en ce temps de bon climat ». «Dix mètres en bas, la rivière gueule avec furie, comme un dragon blessé au combat » (ibid. : 40), mais quand l'enfant rouvre les yeux, et grâce à la conduite sûre de l'officier, le pont est «derrière lui ». Arrivé à la frontière malienne, il ne cesse de revoir « l'image de cette eau furieuse, du pont suspendu au-dessus d'elle » (ibid. : 41).

Dans le village malien où il retrouve sa grand-mère et où on lui fait pourtant fête, il faut que cette dernière lui parle dans un «soussou sans accent » pour que la «connexion à la terre qui l'a vu naître » se rétablisse, et dissiper ainsi « les premières affres de l'exil » (ibid. : 44). Plus tard, alors qu'il voyait en Dakar un Eldorado, la barrière linguistique lui révèle qu'il lui faudra du temps et des efforts pour s'y acclimater. Quant à l'histoire familiale de Maam Matty, elle exprime l'intensité de circulations régionales oublieuses de toute frontière : née à Conakry, avant l'indépendance, de parents sénégalais, elle a épousé un commerçant qui lui a fait faire le voyage inverse de celui de sa fille, de Conakry à Dakar, et l'une de ses jeunes sœurs réside à Bamako. La frontière proprement dite entre les deux États est d'ailleurs franchie sans embûches particulières par Amidou et son protecteur. Bien que ses liens familiaux diasporisés s'étendent à travers toute la sous-région, le sentiment d'étrangeté et d'exil vécu par l'enfant provient avant tout des langues nouvelles qu'il entend. C'est donc le passage d'une vie à une autre, plutôt que d'un pays à l'autre, que traduit celui de la rivière démontée.

Tout en rappelant la proximité géographique et culturelle des pays, la multiplicité des liens, familiaux, amicaux, etc. qui les joignent, l'amour protecteur de la grand-mère dont bénéficie le jeune héros, le narrateur de Destinée ne cesse ainsi de souligner que celui-ci vit pourtant un exil profond, qu'il ne surmontera qu'avec difficultés. Sous des dehors que sa condition sociale privilégiée fait paraître parfois futiles, la narratrice d'AT ne s'en confronte pas moins, elle aussi, à des enjeux fondamentaux, qu'il s'agisse des deuils subis, de ses propres exils « au gré des bourrasques des affectations et des zéphirs de l'espérance » (AT: 158) ou du devenir de la société sénégalaise, redressée à ses yeux après avoir tiré les leçons du Joola, mais où « l'émigration s'est aggravée » (ibid. : 159). Le sort de Max (FE) montre un voyage qui est perdition, un retour rédempteur, et l'on n'a pas de mal à saisir que la jeune Ouly ( $R A)$ joue — et perd — sa vie dans le parcours migratoire qui la conduit à Dakar. Aussi chacune de ces œuvres montre-t-elle 
que si les récits de migration Sud-Sud ne mobilisent que ponctuellement, avec une intensité égale, l'imaginaire tragique déployé par nombre de récits référés aux migrations Sud-Nord, ils ne révèlent pas moins — et il n'y a rien là qui puisse étonner — des enjeux existentiels d'une hauteur équivalente pour les sujets.

La confrontation, dans des œuvres classiques, des scènes d'arrivée dans deux capitales, Dakar et Paris, avait permis de percevoir leur équivalence sémantique. Celle-ci peut se comprendre à partir du rôle de catalyseur que remplissent ces villes pour les destins migrants. Aussi faut-il établir une distinction entre les capitales ouest-africaines dépeintes dans notre corpus contemporain, de simples villes, certes dotées d'une personnalité propre, mais ne représentant que des étapes dans les trajectoires migrantes, et Dakar, quant à elle authentique cosmopolis. La «ville-monde » forme un nœud de virtualités tendues vers l'ailleurs. Elle constitue aussi un lieu d'imbrication entre les rêves des migrants de l'intérieur et les imaginaires circulant à un niveau plus global, grâce aux diasporas qui s'y rencontrent, puis se redéploient depuis les réseaux de circulation qui la traversent. Tout comme il y a une cinquantaine d'années, mais autrement, Dakar connecte, dans les imaginaires innervant les œuvres littéraires, les destins migrants intérieurs à l'Afrique au reste du monde : il y avait auparavant superposition des capitales de l'AOF et de l'Empire ; à présent, la cosmopolis de l'Extrême-Occident africain redistribue et diffuse vers l'extérieur une conscience diasporique inscrite, par les mobilités antérieures, dans la presqu'île elle-même.

Laboratoire Lettres, Langages et Arts. Création, Recherche, Émergence, en Arts, Textes, Images, Spectacles (LLA-Créatis), Université de Toulouse Le Mirail, Toulouse.

\section{BIBLIOGRAPHIE}

BALANDiER, G.

1957 Afrique ambiguë, Paris, Plon («Terre Humaine Poche»).

BREDELOUP, S.

2008 «L'Aventurier, une figure de la migration africaine», Cahiers internationaux de sociologie, CXXV : 281-306.

CAmara, L.

1953 L'Enfant noir, Paris, Plon (« Pocket»).

Coutinho, A.-P., Domingues de Almeida, J. \& Outeirinho, M.-F. (Dir.)

2012 Passages et naufrages migrants. Les fictions du détroit, Paris, L'Harmattan («Études littéraires maghrébines »). 
DADIÉ, B.-B.

2002 [1953] Climbié, in Légendes et poèmes, Abidjan, NEI.

Diome, F.

2003 Le Ventre de l'Atlantique, Paris, Anne Carrière.

GARNIER, X.

2012 «Intellectuels africains en exil à Paris », in X. GARnIER \& J.-P. WARREN (dir.), Écrivains francophones en exil à Paris. Entre cosmopolitisme et marginalité, Paris, Karthala (« Lettres du Sud») : 109-124.

Genette, G.

1982 Palimpsestes. La littérature au second degré, Paris, Éditions du Seuil («Points Essais »).

GLissant, É.

1997 Traité du Tout-Monde. Poétique IV, Paris, Gallimard (« NRF )).

Gueye, M.

2011 La Malédiction de Raabi, Abidjan, NEI-Ceda.

Halen, P.

2001 «Constructions identitaires et stratégies d'émergence : notes pour une analyse institutionnelle du système littéraire francophone », in J. Semujanga (dir.), La Littérature africaine et ses discours critiques, Numéro spécial, Études françaises, 37 (2) : 13-32.

LEIRIS, M.

1955 Fourbis. La règle du jeu II, Paris, Gallimard (« NRF »).

LOPES, B.

1990 [1936] Chiquinho, traduit du portugais par M. Laban, Arles, Actes SudUnesco.

Malaquais, D.

2006a «Cosmopolis : de la ville, de l'Afrique et du monde», Politique Africaine, $100: 7-11$.

2006b «Imaginaires de l'urbain en Afrique aujourd'hui », Politique Africaine, 100 : 17-37.

Mazauric, C.

2012 Mobilités d'Afrique en Europe : récits et figures de l'aventure, Paris, Karthala («Lettres du Sud»).

Mbembe, A.

2006 «Afropolitanisme», Africultures, $<\mathrm{http}: / / \mathrm{www}$. africultures.com/php/index.php?nav=article\&no=4248>.

Ndoye, M.

2007 D'Abidjan à Tunis, Tunis, chez l'auteur. 
PARAVy, F.

2001 «L'Altérité comme enjeu du champ littéraire africain», in R. FonKOUA \& P. Halen (dir.), Les Champs littéraires africains, Paris, Karthala : 213-228.

SADJI, A.

1958 Maïmouna, Paris, Présence Africaine.

Saleh, K., Dahou, K., Nachtman, Y. \& Ndiaye, E. H. S.

1998 Farafina Express, Dakar, ENDA-éditions.

SAMB, M.

2008 Le Regard de l'aveugle, Dakar, Edisal.

SocÉ, O.

1948 Karim, roman sénégalais, Paris, Nouvelles Éditions Latines.

1964 [1937] Mirages de Paris, Paris, Nouvelles Éditions Latines.

VOLTAIRE

1949 [1775] Romans et contes, Paris, Garnier.

YANSANÉ, A.

2010 Destinée. Le récit personnel d'un voyage forcé, Fenton, Éditions Phoenix.

\section{RÉSUMÉ}

On montre, à partir de quatre récits littéraires contemporains (1998-2010), fictionnels ou autobiographiques, de migrations ouest-africaines, et de leur confrontation avec des œuvres classiques (1937-1958) de la littérature africaine francophone, que le passage par Dakar joue, pour la mobilité ouest-africaine, un rôle catalyseur, analogue à celui joué par Paris dans les récits d'immigration. Après I'analyse du tissage des trajectoires migratoires avec les parcours biographiques, est abordée la représentation de la ville comme nœud spatio-symbolique de virtualités, et interface entre imaginaire migratoire régional et conscience diasporique. Enfin, la présence de motifs allégoriques, réactivés par la narration de tout parcours migratoire, souligne que les récits de migration Sud-Sud rendent compte, au même titre que les récits d'émigration SudNord, d'enjeux existentiels fondamentaux pour les sujets.

\section{ABSTRACT}

Books of Passageways. Migratory Paths to and from Dakar. - Through the comparison between four contemporary West African migration narratives (1998-2010), fictional or autobiographical, and a number of now classic francophone African literary works (1937-1958), this paper argues that the passage through Dakar acts as a catalyst for West-African mobility, similar to the role played by Paris in immigration narratives. This article first analyses how migration trajectories are woven into biographies, before going on to explore how the city is depicted both as a spatial and symbolic locus of possibilities, and as an interface between regional imagined worlds 
and diasporic consciousness. Finally, the very presence of allegorical motifs, which are ever revived in the narration of migration itineraries, sets into focus the fact that in South-South migration narratives, just as much as in South-North emigration narratives, fundamental existential issues are at stake.

Mots-clés/Keywords: Dakar, aventure migratoire, cosmopolitisme, exil, imaginaire urbain, littérature/Dakar, migration adventures, cosmopolitanism, urban imagination, exile, literature. 